\title{
Efeito do "fluxo sazonal mínimo da inundação" sobre a composição e estrutura de um campo inundável no Pantanal de Poconé, MT, Brasil
}

\author{
Luciana Rebellato ${ }^{1,3}$ e Cátia Nunes da Cunha ${ }^{2}$
}

Recebido em 29/10/2004. Aceito em 15/04/2005

\begin{abstract}
RESUMO - (Efeito do "fluxo sazonal mínimo da inundação" sobre a composição e estrutura de um campo inundável no Pantanal de Poconé, MT, Brasil). O objetivo deste estudo foi investigar o efeito da sazonalidade hídrica sobre a composição e a estrutura de um campo inundável. Foram utilizadas 30 parcelas (armações de $0,5 \mathrm{~m} \times 0,5 \mathrm{~m}$ ) para as coletas de cada período (chuvoso eestiagem), onde estimou-se o grau de cobertura das espécies. Calculou-se alguns parâmetros fitossociológicos, índices de diversidade e similaridade entre os dois períodos. Foram amostradas 48 espécies, em 35 gêneros, distribuídos em 20 famílias. As famílias com o maior número de espécies foram Cyperaceae (10), Poaceae (seis) e Euphorbiaceae (cinco). As terófitas destacaram-se com 41,02\% e hemicriptófitas com 23,08\%. A diversidade e a riqueza foram maiores no período chuvoso $\left(\mathrm{S}=41, \mathrm{H}^{\prime}=4,01\right.$ e $\left.\mathrm{D}=0,91\right)$ em relação ao período de estiagem $(\mathrm{S}=26$, $\mathrm{H}^{\prime}=3,29$ e D = 0,85), sendo a similaridade igual a $\mathrm{S}_{\mathrm{a}}=55,88 \%$. Algumas espécies como Bacopa myriophylloides Wettst., Cyperus haspan L. ssp. juncoides (Lam.) Kük., Digitaria fuscescens (J. Presl) Henrard, Eleocharis mínima Kunth, foram indicadoras de umidade, enquanto Euphorbia thymifolia L., Wedelia brachycarpa Baker, Ludwigia octovalvis (Jacq.) P.H. Raven, Hydrolea spinosa L., de estiagem. Foram 12 espécies que caracterizaram o campo entre os dois períodos, destacando-se com altos valores de cobertura relativa (CRi) e freqüência relativa (FRi) com variações destes parâmetros entre os dois períodos.
\end{abstract}

Palavras-chaves: Pantanal, campos, estrutura fitossociológica

\begin{abstract}
Minimum seasonal flow" effects on the composition and structure of a flooding grassland in Pantanal of Poconé, Mato Grosso do Sul State, Brazil). The aim of this study was to investigate the seasonal effect of wet and dry periods on the composition and structure of flooding grassland. Thirty quadrats $(0,5 \mathrm{~m} \times 0,5 \mathrm{~m})$ were used in each period (wet and dry) to estimate the degree of covering of the species. Phytosociological parameters, diversity and similarity indices were calculated between periods. Forty eight species were collected, 35 genera distributed in 20 families. Cyperaceae (10), Poaceae (six) and Euphorbiaceae (five) were the families with the highest number of species. Therophytes were the most abundant (41,02\%), followed by hemicryptophytes (23,08\%). Diversity and richness were higher in wet season $\left(\mathrm{S}=41, \mathrm{H}^{\prime}=4,09 \mathrm{e} \mathrm{D}=0,91\right)$ than in the dry season $(\mathrm{S}=26, \mathrm{H}=3,29 \mathrm{e} \mathrm{D}=0,85)$, and the similarity was $\mathrm{S}_{\mathrm{s}}=55,88 \%$. Some species, like Bacopa myriophylloides Wettst, Cyperus haspan L. ssp. juncoides (Lam.) Kük, Digitaria fuscescens (J. Presl) Henrard and Eleocharis minima Kunth, were humidity indicators, while Euphorbia thymifolia L., Wedelia brachycarpa Baker, Ludwigia octovalvis (Jacq.) P.H. Raven, Hydrolea spinosa L. were the dry ones. High values of relative covering and relative frequency of 12 species outstood, characterizing the area within both periods, with variation of these parameters between them.
\end{abstract}

Key words: Pantanal, grasslands, phytosociological structure

\section{Introdução}

No alto da bacia do rio Paraguai existem planícies deprimidas que são preenchidas anualmente por deposições aluviais. Essas planícies inundáveis recebem o nome de Pantanal e atuam regulando a hidrologia do rio Paraguai, influenciando o clima, retendo sedimentos e funcionando como filtro biológico de resíduos orgânicos e nutrientes vindos dos afluentes (Junk \&
Silva 1999). Os padrões e processos dos ecossistemas do Pantanal são regulados pelo ciclo anual de inundações e secas (Junk et al. 1989). A distribuição das espécies e a diversidade variam dentro e entre os diferentes hábitats dispostos ao longo do gradiente de inundação, desde os não inundáveis, os sazonal e os permanentemente inundados (Cabido et al. 1996; Nunes da Cunha \& Junk 1999; 2001; Silva et al. 2001). Sarmiento \& Monasterio (1983) usaram o termo

\footnotetext{
Rua 19, Casa 3, Quadra 28, Jardim Universitário, CEP 78075-580, Cuiabá, MT, Brasil

2 Universidade Federal de Mato Grosso, Departamento de Botânica e Ecologia, NEPA- IB/UFMT, Rua Fernando Corrêa e Costa s/n, Bairro Coxipó, CEP 78060-900, Cuiabá, MT, Brasil

3 Autor para correspondência: lurebellato@ hotmail.com
} 
"savanas hipersazonais" para descrever esse tipo de vegetação sujeito à inundação sazonal.

Entre as diversas fitofisionomias do Pantanal estão os campos nativos sazonalmente inundáveis que são utilizados para a pecuária extensiva. A fisionomia campestre está correlacionada ao meso-relevo e intensidade da inundação sazonal (Cunha 1980) e, freqüentemente, é dominada por uma única espécie de gramínea (Pott 1988).

A inundação é um evento natural que promove mudanças na estrutura e composição florística nessas comunidades (Prado et al. 1994; Schessl 1999). A manutenção da diversidade de espécies, frente a diferentes condições ambientais, tem sido alvo de estudos recentes nas áreas da ecologia de comunidades e conservação biológica (Grime 1979; Maarel 1993; Palmer 1994; Kiehl et al. 1996). Esse conhecimento é de fundamental importância para o manejo dos campos nativos no Pantanal (Allem \& Valls 1987). No entanto, são poucos os trabalhos referentes a composição e estrutura dos campos nativos sazonalmente inundáveis no Pantanal, principalmente os que avaliam as mudanças influenciadas pelo pulso inundação (Prado et al. 1994; Pott \& Adamoli 1999; Schessl 1999; Crispim et al. 2002; Nogueira et al. 2002; Pozer \& Nogueira 2004; L. Rebellato, dados não publicados).

O presente trabalho tem como objetivo analisar as mudanças na composição florística e estrutura de um campo inundável no Pantanal de Mato Grosso, considerando o efeito do regime hidrológico sobre a vegetação. Os resultados permitem ampliar o conhecimento sobre a ecologia desses ambientes.

\section{Material e métodos}

Área de estudo - O estudo foi conduzido em um campo nativo inundável da fazenda Nossa Senhora Aparecida (16 22'07'S e 56 20'26'W), nordeste do Pantanal de Poconé, município de Nossa Senhora do Livramento, Mato Grosso, próximo à localidade do Pirizal. Este campo nativo inundável é utilizado como pastagem do rebanho bovino no período de estiagem. A vegetação é formada por um estrato herbáceo curtigraminoso dominante (aproximadamente $20 \mathrm{~cm}$ ), esporadicamente entremeado com uma espécie mais alta, Axonopus leptostachyus (Flüeggé) Hitchc. (aproximadamente $80 \mathrm{~cm})$.

O clima é do tipo AW-quente e úmido (Köppen 1948), com precipitação média de $1.250 \mathrm{~mm}$ ao ano e a média anual de temperatura de $25,8^{\circ} \mathrm{C}$. No Pantanal duas estações são observadas durante o ano, sendo uma seca, no período de maio a setembro e a outra chuvosa, de outubro a abril (Nunes da Cunha \& Junk 2004). O processo de inundação sazonal é dividido em quatro fases: enchente, cheia, vazante e seca (Prado et al. 1994).

Ponce (1995) cita cinco tipos de inundação para o Pantanal: mínima, comum, média, extraordinária e excepcional. A variabilidade no fluxo da inundação anual pode ser observada nas médias anuais da série histórica do nível d'água do Rio Paraguai (Fig. 1). Em adição ao fluxo anual de inundação, ocorrem anos seguidos de grandes inundações e de pronunciadas secas, considerados como períodos plurianuais (Nunes da Cunha \& Junk 2001) (Fig. 1).

No Pantanal de Poconé a inundação normalmente ocorre entre janeiro e abril, juntamente com a estação chuvosa (Fig. 2). O ano deste estudo (2001) foi mais seco e considerado de "inundação mínima", ou seja, não houve inundação comum, devido a baixa pluviosidade nos meses de dezembro/2000, janeiro e fevereiro/2001 (Fig. 3). Neste ano a pluviosidade dos meses chuvosos não seguiu o padrão clássico (Fig. 2), como pode ser verificado no final do ano de $2001 \mathrm{e}$ início de 2002 (Fig. 3). Durante o período normal de inundação do campo nativo da área de estudo a lâmina d'água pode atingir até $1,20 \mathrm{~m}$ de altura e predominância de plantas aquáticas, mas no ano de estudo alcançou aproximadamente $13 \mathrm{~cm}$. Por este motivo, dividiu-se o período sazonal em duas fases, chuvosa e estiagem, e não em quatro fases. Para a definição dos meses secos e úmidos foram utilizadas as considerações de Rizzini (1997).

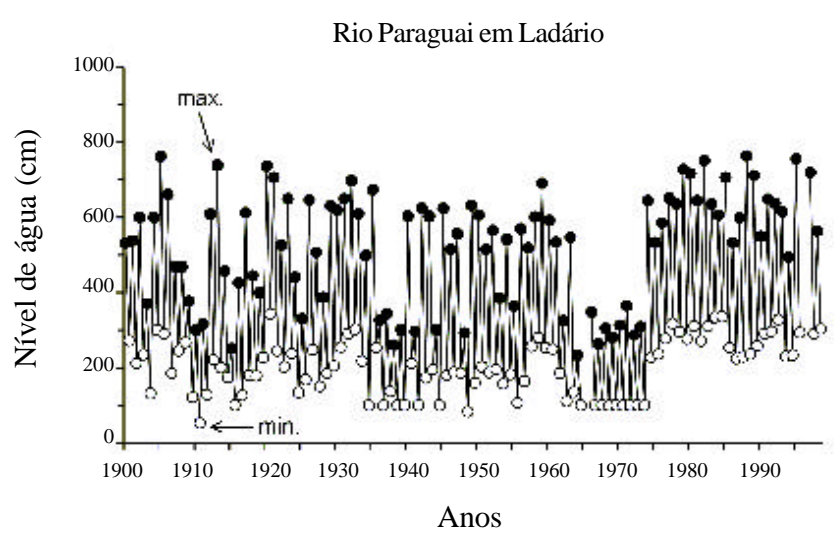

Figura 1. Média anual da flutuação do nível da água do Rio Paraguai, em Ladário, MT, Brasil (1900-1990), com períodos plurianuais de inundações sazonais mínimas (entre os anos de 1935 e 1940 e os anos de 1964 e 1974) e inundações extraordinárias (entre os anos de 1974 e 1990), e anos com fluxos variáveis de inundação. (INEMET). 


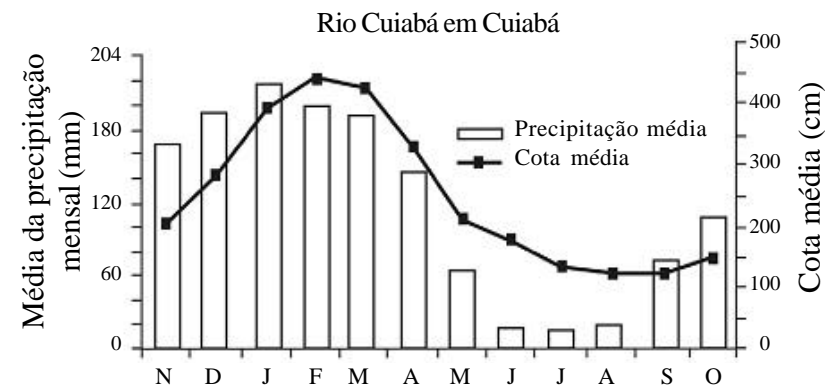

Figura 2. Média anual da flutuação do nível da água do rio Cuiabá (1933-1993) e média da precipitação, próximo a Cuiabá, norte do Pantanal, MT, Brasil (1971-1988) (INEMET). Período chuvoso compreendido entre os meses de outubro a abril e período de seca entre os meses de maio a setembro.

Coleta de dados - Os estudos foram realizados em três meses consecutivos chuvosos (fevereiro, março e abril) da estação chuvosa e em três meses consecutivos de estiagem (julho, agosto e setembro) da estação seca (Fig. 3). A composição das espécies, a sua forma de vida (Raunkier 1934) e o grau de cobertura dos indivíduos foram determinados por amostragens mensais, utilizando-se 10 parcelas não fixas (armações metálicas de 0,5 m×0,5 m), lançadas aleatoriamente no campo nativo. Foram amostradas, portanto, 30 parcelas $\left(7,5 \mathrm{~m}^{2}\right)$ tanto no período chuvoso como no período de estiagem.

Primeiramente, realizou-se a listagem das espécies vegetais presentes na parcela e, posteriormente, foi realizada a estimativa do grau de cobertura (visual e em porcentagem) dos indivíduos de cada espécie, tendo como base a projeção horizontal da espécie sobre o solo do campo (Greig-Smith 1957 apud Kershaw 1978). O grau de cobertura de cada espécie foi estimado independentemente das outras, tanto que ao final, os valores de cobertura de todas as espécies, juntos, pudessem exceder 100\% (Pakarinen 1984). Em cada parcela coletou-se amostra de material botânico, visando identificação taxonômica.

As espécies coletadas foram herborizadas e identificadas por comparações com espécimes da coleção do herbário da Universidade Federal de Mato Grosso (UFMT), por especialistas na flora pantaneira e com bibliografias especializadas (Pott \& Pott 1994; 2000). As amostras foram incorporadas ao acervo do UFMT, Herbário Central.

O sistema de classificação das espécies adotado foi o de Cronquist (1981). Quanto às formas de vida, as espécies foram classificadas de acordo com o sistema de Raunkier (1934), em dados já publicados por Pott \& Adamoli (1999) e Schessl (1999).

Análise dos dados - Com os dados de composição florística e grau de cobertura dos indivíduos foram calculados parâmetros fitossociológicos, estimados índices de diversidade e a similaridade florística entre período chuvoso e estiagem. O grau de cobertura das espécies de cada parcela foi definido utilizando uma escala de cobertura proposta por Braun-Blanquet (1979), para as estimativas dos parâmetros fitossociológicos, sendo: grau de cobertura muito pequeno, cobrindo entre 1 e $10 \%$ da superfície do solo (grau médio de cobertura: 5\%); entre 10 e $25 \%$ de cobertura da superfície do solo (grau médio de cobertura: $17,5 \%$ ); entre 25 e $50 \%$ (grau médio de cobertura: $37,5 \%$ ); entre 50 e $75 \%$ (grau médio de cobertura: 62,5\%); entre 75 e $100 \%$ (grau médio de cobertura: $87,5 \%)$.

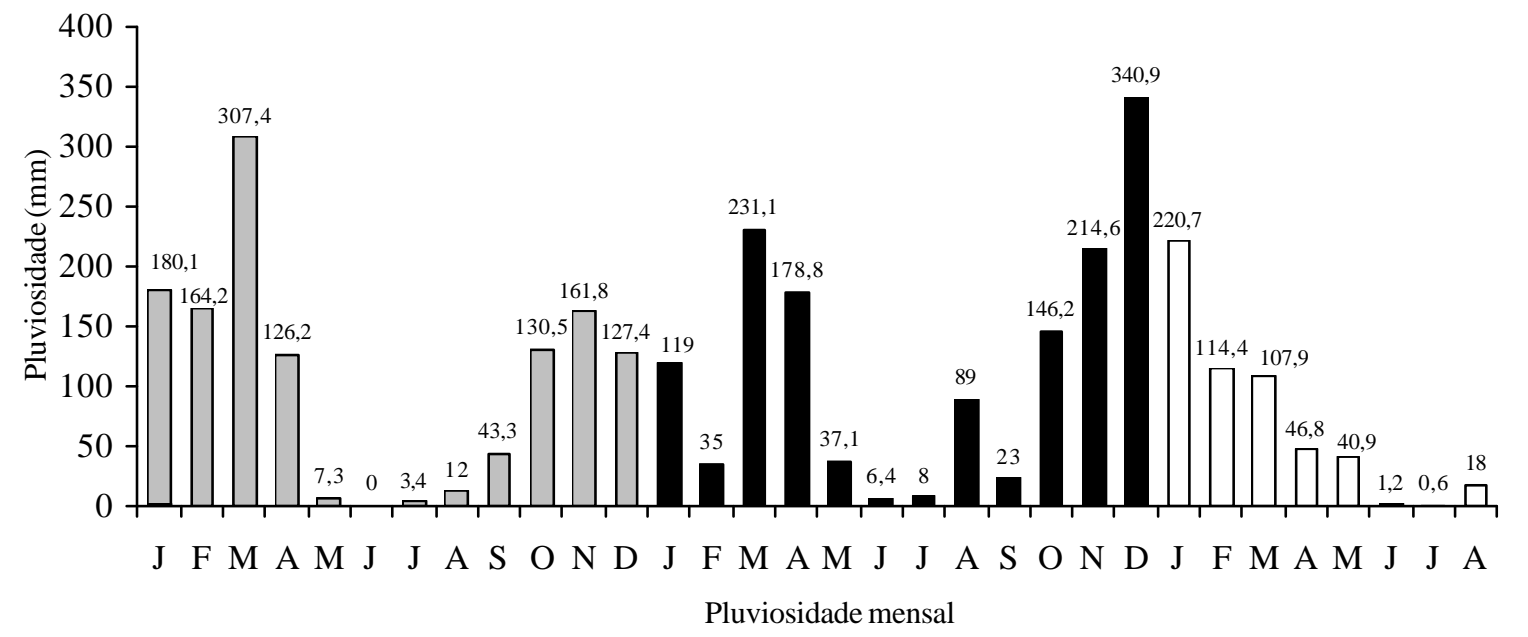

Figura 3. Pluviosidade mensal de 2000, 2001 e 2002 na cidade de Várzea Grande, MT, Brasil (INEMET). Período chuvoso de outubro a abril e período de estiagem de maio a setembro. $\square=2000 ; \mathbf{\square}=2001 ; \square=2002$. 
792 Rebellato \& Nunes da Cunha: Efeito do "fluxo sazonal mínimo da inundação" sobre a composição e estrutura ...

Para o cálculo dos parâmetros fitossociológicos, foram somados os dados referentes aos três meses de coleta consecutivos, representando cada período estudado, ou seja: período chuvoso (fevereiro, março e abril) e período de estiagem (julho, agosto e setembro). Sendo assim, cada estação do ano foi representada por uma coleta geral.

Os parâmetros fitossociológicos estimados para as espécies foram:

$\mathrm{FA}=100 . \mathrm{p} / \mathrm{PT} ; \quad \mathrm{FR}=100 . \mathrm{FA} / \Sigma \mathrm{FA} ;$ $\mathrm{ACi}=\Sigma($ gck.apk/100); VC $=100 . \mathrm{AC} / \mathrm{AT}$, sendo AT $=\Sigma$ apk e $\mathrm{CR}=100 . \mathrm{AC} / \Sigma \mathrm{AC}$

onde:

FA: frequiência absoluta (\%)

FR: freqüência relativa $(\%)$

$\mathrm{AC}$ : área de cobertura $\left(\mathrm{m}^{2}\right)$

VC: valor de cobertura (\%) (Braun-Blanquet 1979)

CR: valor de cobertura relativa (\%) na área total coberta pelo estrato baixo (Barbour et al. 1980)

p: número de parcelas

PT: número total de parcelas $(\mathrm{n}=30)$

gck: grau médio de cobertura $(\%)$

ap: área da parcela $\mathrm{k}\left(\mathrm{m}^{2}\right)$

AT: área total amostrada $\left(\mathrm{m}^{2}\right)$

Para o cálculo da diversidade alfa (Whittaker 1975) foram utilizados as medidas de riqueza de espécies (S), definida como simplesmente o número de espécies numa amostra (Peet 1974), os índices de ShannonWienner $\left(\mathrm{H}^{\prime}=-\Sigma(\mathrm{ni} / \mathrm{N}) \log _{2}(\mathrm{ni} / \mathrm{N})\right)$, que atribui maior peso às espécies raras, e Simpson $\left(\mathrm{D}=\sum(\mathrm{ni}(\mathrm{ni}-1) / \mathrm{N}(\mathrm{N}-\right.$ 1)), que é pouco influenciado por espécies raras e equabilidade (J=H'/Hmáx) (Magurran 1988), onde:

ni=valor de importância (dado pelo $\mathrm{VCi}$ ) para cada espécie

$\mathrm{N}=$ total dos valores de importância para todas as espécies na amostra

Para o cálculo dos índices de diversidade utilizouse o programa MVSP (versão 3.13) (Kovach 1993).

A similaridade florística entre os períodos chuvoso e de estiagem foi calculada pelo coeficiente de Sorensen $\left(\mathrm{S}_{\mathrm{s}}=2 \mathrm{a} / 2 \mathrm{a}+\mathrm{b}+\mathrm{c}\right)($ Krebs 1985), onde:

a: número de espécies comuns aos dois períodos

b: número de espécies no período chuvoso, mas não no período de estiagem

c: número de espécies no período de estiagem, mas não no período chuvoso

Uma classificação por TWINSPAN ("Two-way indicator species analysis") foi realizada para identificar espécies preferenciais dos dois períodos (chuvoso e de estiagem) e as indiferentes (Hill 1979).

\section{Resultados e discussão}

Foram encontradas 48 espécies e 35 gêneros distribuídos em 20 famílias (Tab. 1). Todas as espécies estão citadas em Pott \& Pott (1999) para a flora atual do Pantanal. As famílias com o maior número de espécies são Cyperaceae (10), Poaceae (seis) e Euphorbiaceae (cinco). Algumas espécies foram identificadas apenas ao nível de gênero (cinco espécies) ou família (uma espécie) devido à rara ocorrência e por se encontrarem em estágio vegetativo. Nunes da Cunha et al. (2001), em trabalho em áreas de campo e de brejo próximos a área do presente estudo, inventariaram 64 espécies, sendo Poaceae a família com maior número de espécies (10), seguida de Cyperaceae (cinco), Convolvulaceae e Euphorbiaceae (quatro). Pott \& Adamoli (1999) registraram a família Poaceae como a mais rica com 65 espécies para várias comunidades do Pantanal de Paiaguás, MS, seguida de Leguminosae (44), Rubiaceae (14), Asteraceae (12), e Cyperaceae e Euphorbiaceae (10). Schessl (1999), em várias comunidades do Pantanal de Poconé, MT, também registrou Poaceae como a mais rica em espécies (104), seguida de Leguminosae (57) e Cyperaceae (56). Schessl (1999) comparando seus resultados do Pantanal com oito regiões alagáveis da América do Sul, verificou baixa similaridade ao nível de espécie, mas considerável similaridade ao nível de família. Segundo o autor, Poaceae, Leguminosae (lato senso) e Cyperaceae são as famílias que concentraram maior número de espécies nessas áreas. Portanto, Poaceae e Cyperaceae são as famílias mais ricas da vegetação campestre, entretanto, quando analisa-se sob o ponto de vista de várias comunidades no Pantanal soma-se a estas a família Leguminosae (lato senso) como uma das mais ricas.

Prado et al. (1994) comentaram que, provavelmente, a família Cyperaceae é a mais rica em macrófitas no Pantanal. No presente estudo essa família apresentou maior número de espécies durante o período chuvoso (10 espécies) em relação ao período de estiagem (três espécies). Crispim et al. (2002) também verificaram aumento na dispersão das Cyperaceae (nove espécies) após inundação, em campos do Pantanal entre as subregiões de Abobral e Nhecolândia (MS), e nos meses de seca maior participação das Poaceae. O número de espécies aparentemente inferior aos demais trabalhos citados se justifica por estarmos estudando apenas um tipo de campo, enquanto que os outros abordaram diferentes formações campestres, além de outras fisionomias. Portanto, proporcionalmente o número de 
Tabela 1. Valores de riqueza de espécies (S), índices de diversidade de Shannon-Wiener (H') e Simpson (D), Equabilidade (E), listagem de famílias e espécies com respectivos valores de cobertura relativa (CR) e freqüência relativa (FR), encontradas no período chuvoso e de estiagem no campo inundável na Fazenda Nossa Senhora Aparecida, Pantanal de Poconé, MT, Brasil. Hemi = hemicriptófita, Geo = geófita, Tero $=$ terófita, Fane $=$ fanerófita, Came $=$ caméfita, $\mathrm{Ni}=$ não identificada.

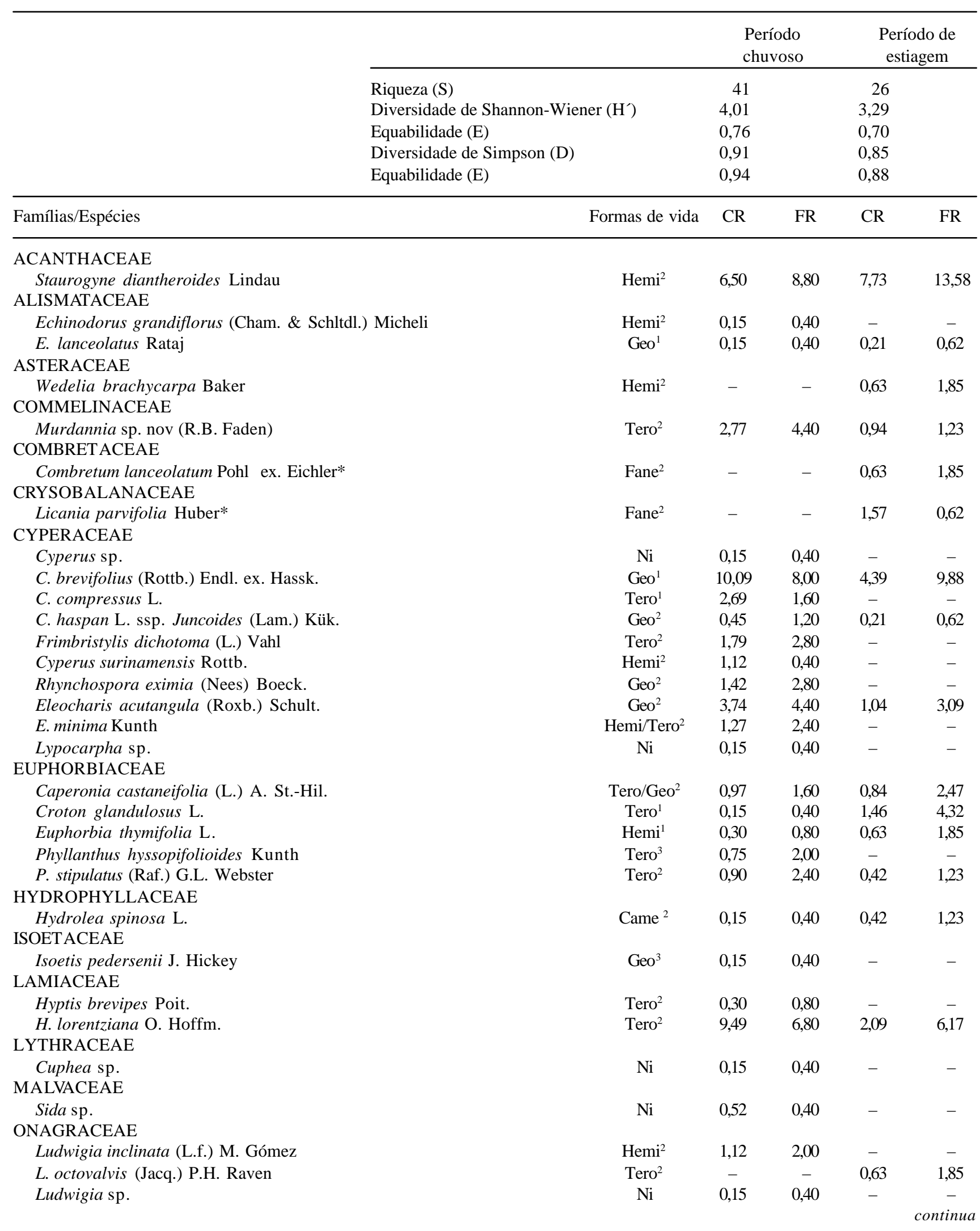


794 Rebellato \& Nunes da Cunha: Efeito do "fluxo sazonal mínimo da inundação" sobre a composição e estrutura ...

Tabela 1 (continuação)

\begin{tabular}{|c|c|c|c|c|c|}
\hline \multirow[b]{2}{*}{ Famílias/Espécies } & \multirow[b]{2}{*}{ Formas de vida } & \multicolumn{2}{|c|}{$\begin{array}{l}\text { Período } \\
\text { chuvoso }\end{array}$} & \multicolumn{2}{|c|}{$\begin{array}{l}\text { Período de } \\
\text { estiagem }\end{array}$} \\
\hline & & $\mathrm{CR}$ & FR & $\mathrm{CR}$ & FR \\
\hline \multicolumn{6}{|l|}{ POACEAE } \\
\hline Axonopus leptostachyus (Flüeggé) Hitchc. & Hemi/Came $^{2}$ & 0,30 & 0,80 & 8,88 & 4,94 \\
\hline Digitaria fuscescens (J. Presl) Henrard & $\mathrm{Hemi}^{2}$ & 0,45 & 1,20 & - & - \\
\hline Eragrostis rufescens Schrad ex. Schult. & $\mathrm{Ni}$ & 0,15 & 0,40 & 0,21 & 0,62 \\
\hline Panicum laxum Sw. & Tero $^{2}$ & 6,05 & 8,00 & 28,21 & 14,20 \\
\hline Reimarochloa brasiliensis (Spreng.) Hitchc. & Tero $^{1}$ & 14,87 & 7,20 & 13,06 & 7,41 \\
\hline Setaria geniculata P. Beauv. & $\mathrm{Hemi}^{1}$ & 3,21 & 4,00 & 7,42 & 6,79 \\
\hline \multicolumn{6}{|l|}{ PONTEDERIACEAE } \\
\hline Pontederia parviflora Alexander & $\mathrm{Ni}$ & 1,57 & 1,60 & - & - \\
\hline \multicolumn{6}{|l|}{ PORTULACACEAE } \\
\hline $\begin{array}{l}\text { Portulaca fluvialis Legrand } \\
\text { RUBIACEAE }\end{array}$ & \multicolumn{4}{|c|}{ RUBIACEAE } & - \\
\hline Diodia kuntzei K. Schum. & Tero $^{2}$ & 17,19 & 8,80 & 17,35 & 10,49 \\
\hline Richardia grandiflora (Cham. \& Schltdl.) Steud. & Tero $^{2}$ & 0,15 & 0,40 & - & - \\
\hline Sipanea biflora (L.f.) Cham. \& Schltdl. & $\mathrm{Hemi}^{1}$ & 2,54 & 1,60 & - & - \\
\hline Rubiaceae sp. & $\mathrm{Ni}$ & 0,15 & 0,40 & - & - \\
\hline \multicolumn{6}{|l|}{ SCROPHULARIACEAE } \\
\hline Bacopa myriophylloides Wettst. & Tero $^{2}$ & 4,71 & 6,40 & - & - \\
\hline B. salzmannii (Benth.) Wettst. ex Edwall & $\mathrm{Ni}$ & 0,97 & 1,60 & 0,21 & 0,62 \\
\hline B. aff. verticillata (Penn. \& Gl.) Penn. & Tero $^{1}$ & - & - & 0,21 & 0,62 \\
\hline \multicolumn{6}{|l|}{ STERCULIACEAE } \\
\hline Melochia arenosa Benth. & $\mathrm{Came}^{2}$ & - & - & 0,42 & 1,23 \\
\hline M. parvifolia Kunth & $\mathrm{Came}^{2}$ & - & - & 0,21 & 0,62 \\
\hline Totais & & $100 \%$ & $100 \%$ & $100 \%$ & $100 \%$ \\
\hline
\end{tabular}

${ }^{1}$ Classificação das formas de vida de acordo com Pott \& Adámoli (1996); ${ }^{2}$ Classificação das formas de vida de acordo com Schessl (1999); ${ }^{3}$ Classificação das formas de vida de acordo com Pott \& Pott (2000); *Plântulas

espécies obtido no presente estudo é alto.

Formas de vida - As espécies terófitas destacaramse com 41,02\% (16) e as espécies hemicriptófitas com $23,08 \%$ (nove), seguidas das geófitas com $15,38 \%$ (seis), das caméfitas com 7,69\% (três), e das fanerófitas com $5,13 \%$ (duas). Algumas espécies possuem duas formas de vida dependente da situação ambiental, sendo $2,63 \%$ (uma) tanto hemicriptófita como terófita, 2,56\% (uma) tanto terófita como geófita e $2,56 \%$ (uma) tanto hemicriptófita como caméfita (Tab. 1). As espécies terófitas predominaram em ambas as fases do ano, entretanto, as terófitas juntamente com as hemicriptófitas também foram características do período chuvoso (Tab. 2). As terófitas e hemicriptófitas predominaram nas áreas de campo e brejo estudadas por Nunes da Cunha et al. (2001) no Pantanal de Poconé, com 36 e 30\% respectivamente. As espécies terófitas e hemicriptófitas foram associadas por Pott \& Adámoli (1999) com o gradiente de inundação crescente, isto é, às áreas sazonalmente inundadas, tendo as fanerófitas tendência inversa. Para Sarmiento \& Monastério (1983) o alto valor de hemicriptófitas evidenciou uma característica típica de formações campestres savânicas de áreas tropicais. Este predomínio de terófitas indica a importância do banco de sementes para a manutenção destas comunidades e a não resistência ao manejo pelo fogo.

A riqueza de espécies e os índices de diversidade de Shannon-Wienner e Simpson foram maiores no período chuvoso $\left(\mathrm{S}=41, \mathrm{H}^{\prime}=4,01\right.$ nats/ind. e

Tabela 2. Quantificação das espécies quanto às formas de vida ocorrentes no campo inundável em ambos os períodos, apenas no período chuvoso e no período de estiagem, e respectivos valores totais e percentuais. Fazenda Nossa Senhora Aparecida, Pantanal de Poconé, MT, Brasil.

\begin{tabular}{lcccrr}
\hline Forma de vida & $\begin{array}{c}\text { Ambas } \\
\text { as fases }\end{array}$ & Chuvoso Estiagem & Total & $\%$ \\
\hline Terófita & 7 & 7 & 2 & 16 & 41,02 \\
Hemicriptófita & 3 & 5 & 1 & 9 & 23,08 \\
Geófita & 4 & 2 & 0 & 6 & 15,38 \\
Caméfita & 1 & 0 & 2 & 3 & 7,69 \\
Fanerófita & 0 & 0 & 2 & 2 & 5,13 \\
Terófita/geófita & 1 & 0 & 0 & 1 & 2,56 \\
Hemicriptófita/caméfita & 1 & 0 & 0 & 1 & 2,56 \\
Hemicriptófita/terófita & 0 & 1 & 0 & 1 & 2,56 \\
& & & & &
\end{tabular}


$\mathrm{D}=0,91$ nats/ind.) em relação ao período de estiagem ( $\mathrm{S}=26, \mathrm{H}^{\prime}=3,29$ nats/ind. e $\mathrm{D}=0,85$ nats/ind.); a média dos índices de Shannon-Wienner entre os dois períodos é de 3,69 nats/ind. Sala et al. (1986), em estimativa da média anual da diversidade no pampa inundável argentino, da província de Buenos Aires, encontraram $H^{\prime}=3,3$ nats/ind. Prado et al. (1994), ao contrário do que foi observado neste estudo, verificaram queda da riqueza de espécies correlacionada com o aumento do nível d'água em uma área no Pantanal de Poconé, MT, aparentemente em um período de inundação comum. $\mathrm{O}$ alto valor dos índices de diversidade durante o período chuvoso provavelmente está relacionado com a baixa intensidade da inundação, o que permitiu tanto o estabelecimento de espécies anfíbias e aquáticas, quanto a permanência de algumas espécies terrestres que puderam suportar tal intensidade de estresse hídrico. Portanto, é possível que dependendo da intensidade e duração da perturbação haja diferentes respostas das comunidades campestres com relação à riqueza e diversidade.

A similaridade florística entre os dois períodos foi alta $(55,88 \%)$, de acordo com o coeficiente de Sørensen. Para o índice de Sørensen, quanto mais próximo de $100 \%$, maior a similaridade, e uma similaridade maior que $50 \%$ pode ser considerada alta (Kent \& Coker 1994). Do total das espécies amostradas, 19 ocorreram em ambos períodos, 22 apenas no período chuvoso e sete espécies apenas no período de estiagem. Próximo a este índice foi o encontrado por Prado et al. (1994), quando compararam as fases cheia e seca (ISS $=55 \%$ ).

A classificação feita por Twinspan separou os meses em dois grupos principais (0 e 1), sendo o autovalor igual à 0,25 , considerado baixo. Este autovalor baixo ocorreu devido ao grande número de espécies não preferenciais, ou de ampla distribuição (33 espécies). O grupo de espécies relacionadas a maior umidade (grupo 0), formado pelos meses de fevereiro, março e abril, tiveram como espécies indicadoras Bacopa myriophylloides, Cyperus haspan, Digitaria fuscescens, Eleocharis minima, Cuphea sp., Murdannia sp., Phyllanthus hyssopifolioides, Phyllanthus stipulatus, Pontederia parvifolia e Sipania biflora (Fig. 4). Com exceção de Digitaria fuscescens e Sipania biflora todas as outras espécies foram citadas por Pott \& Pott (2000) e Pott et al. (1999) como plantas aquáticas. $\mathrm{O}$ grupo 1 , formado pelos meses de julho, agosto e setembro, têm como espécies indicadoras Euphorbia thymifolia, Wedelia brachycarpa, Ludwigia octovalvis, Hydrolea spinosa e Combretum lanceolatum, que podem ocorrer tanto em ambientes mais úmidos quanto nos mais secos.

As dez espécies que se destacaram quanto a cobertura relativa (CR) durante o período chuvoso somam $78,62 \%$, sendo $25,04 \%$ desta cobertura correspondente às gramíneas. Dentre estas dez espécies, Diodia kuntzei (17,19\%) e Reimarochloa brasiliensis $(14,87 \%)$ aparecem com os maiores valores (Fig. 5).

As dez espécies que se destacaram com os maiores valores de cobertura relativa (CR) no período de estiagem somam $91,64 \%$, só gramíneas participam com $57,78 \%$. Valor similar foi verificado por Schessl (1999), durante a fase seca, nas fazendas Nova Berlim e Ipiranga, Pantanal de Poconé. Com referencia às dez espécies, Panicum laxum $(28,21 \%)$ e Diodia kuntzei $(17,35 \%)$ apresentaram os maiores valores de CR (Fig. 6).

Pott (1986) citou Panicum laxum e Reimarochloa brasiliensis como espécies preferenciais de ambientes sazonalmente muito alagados. A espécie de gramínea Panicum laxum teve seu grau de cobertura fortemente ligado ao período chuvoso, mesmo durante este ano de período de inundação mínima. Ao contrário,

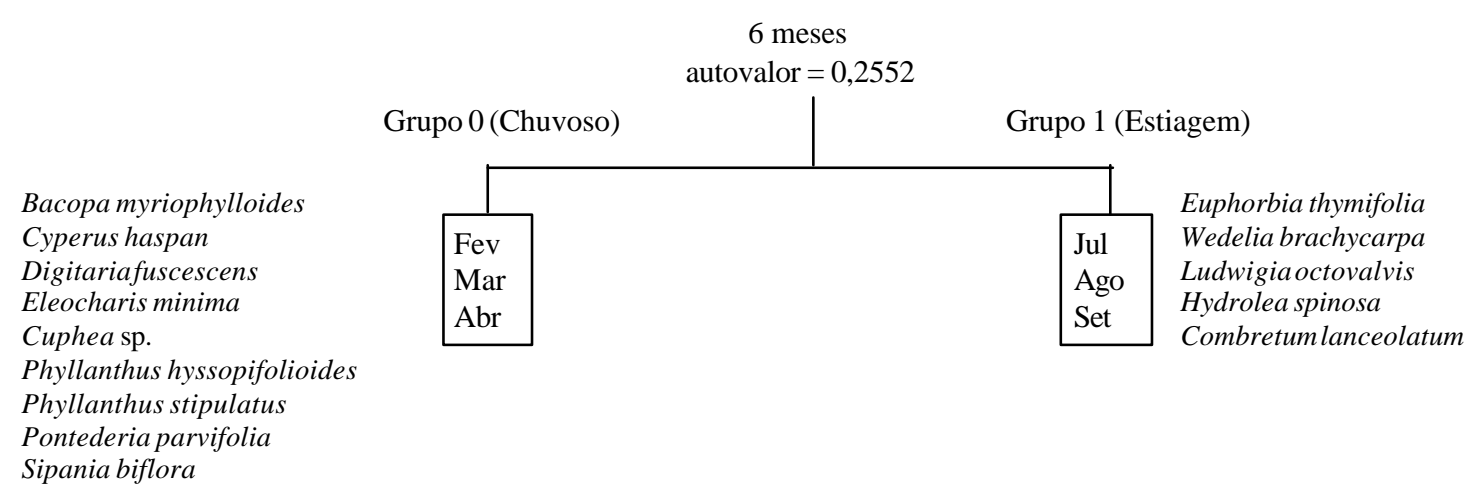

Figura 4. Classificação pelo TWINSPAN dos seis meses de coleta da área de estudo, campo inundável, Fazenda Nossa Senhora Aparecida, Pantanal de Poconé, MT, Brasil. 
Rebellato \& Nunes da Cunha: Efeito do "fluxo sazonal mínimo da inundação" sobre a composição e estrutura ...

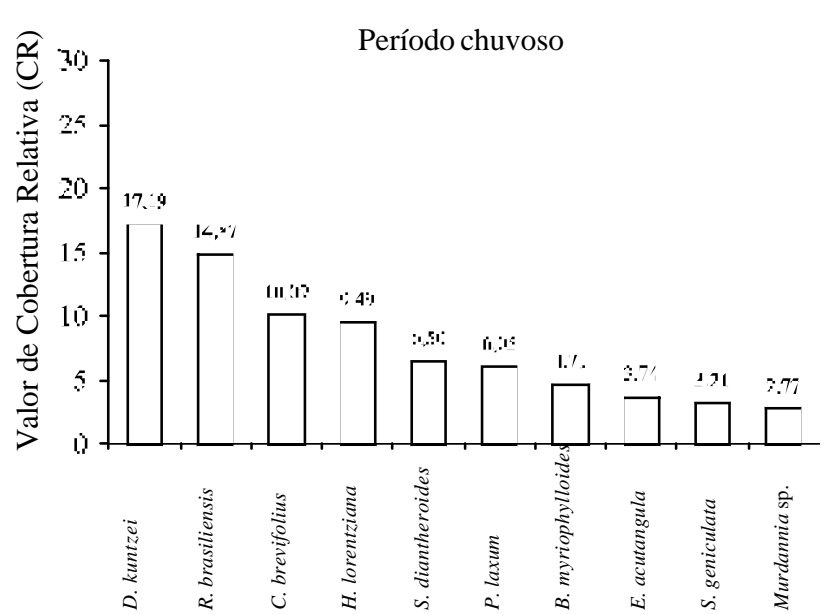

Figura 5. Dez espécies com maiores valores de cobertura relativa (CR) durante o período chuvoso, campo inundável, Fazenda Nossa Senhora Aparecida, Pantanal de Poconé, MT, Brasil.

Reimarochloa brasiliensis se manteve estável em ambos os períodos (Fig. 5 e 6).

Em relação aos dez maiores valores de cobertura relativa, oito espécies ocorreram tanto no período chuvoso, quanto no de estiagem. Como indicadoras de período chuvoso destacaram-se Bacopa myriophilloides e Murdannia sp. Enquanto que Axonopus leptostachyus e Croton glandulosus indicaram o período de estiagem (Fig. 5 e 6). Esta última espécie tem seu aumento relacionado aos anos mais secos de acordo com Pott \& Pott (1994).

Os valores de freqüência relativa evidenciaram praticamente as mesmas espécies anteriormente citadas. As espécies que apresentaram maiores valores de frequiência relativa durante o período chuvoso foram Diodia kuntzei $(8,80 \%)$, Staurogyne diantheroides

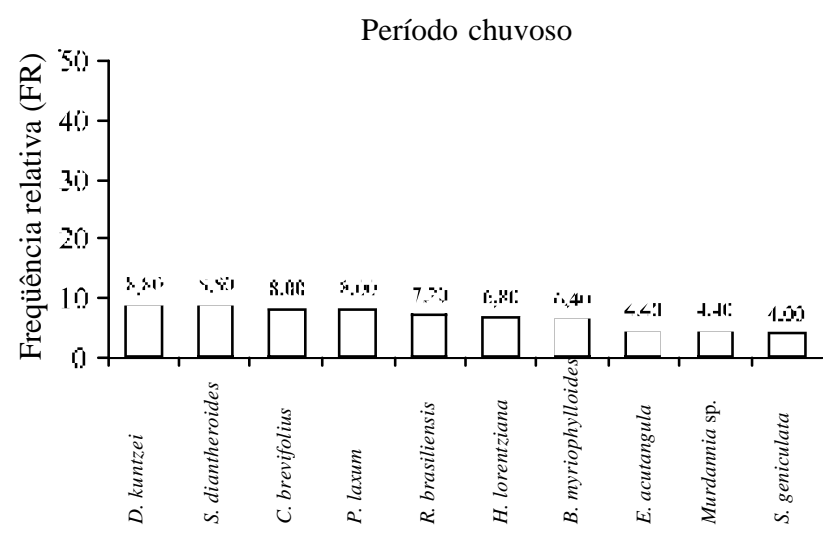

Figura 7. Dez espécies com maiores valores de frequiência relativa (FR) durante o período chuvoso, campo inundável, Fazenda Nossa Senhora Aparecida, Pantanal de Poconé, MT, Brasil.

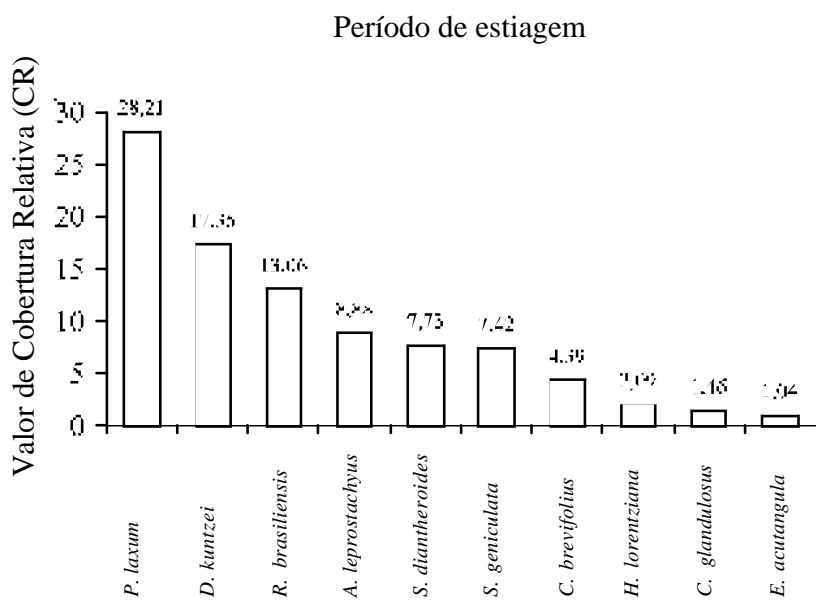

Figura 6. Dez espécies com maiores valores de cobertura relativa (CR) durante o período de estiagem, campo inundável, Fazenda Nossa Senhora Aparecida, Pantanal de Poconé, MT, Brasil.

(8,80\%), Cyperus brevifolius (8\%) e Panicum laxum $(8 \%)$. Entre as que apresentaram maiores valores de freqüência relativa no período de estiagem estão Panicum laxum (14,2\%), Staurogyne diantheroides $(13,58 \%)$, Diodia kuntzei $(10,49 \%)$ e Cyperus brevifolius $(9,88 \%)$ (Fig. 7 e 8$)$.

A variação nos valores de cobertura relativa também foi observada para Cyperus brevifolius, Setaria geniculata, Hyptis lorentziana e Eleocharis acutangula entre os dois períodos (Fig. 9).

Quanto à amplitude de distribuição das espécies, Pott \& Adámoli (1999) citaram Croton glandulosus, Setaria geniculata, Panicum laxum e Bacopa myriophylloides ocorrendo em diversas comunidades.

Durante um ano de inundação comum, Schessl (1999) verificou mudança sazonal na composição dos

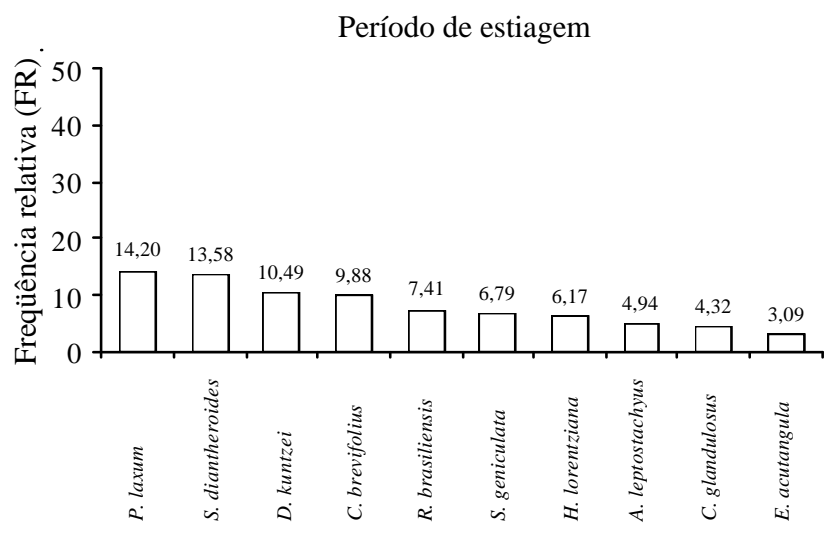

Figura 8. Dez espécies com maiores valores de freqüência relativa (FR) durante o período de estiagem, campo inundável, Fazenda Nossa Senhora Aparecida, Pantanal de Poconé, MT, Brasil. 


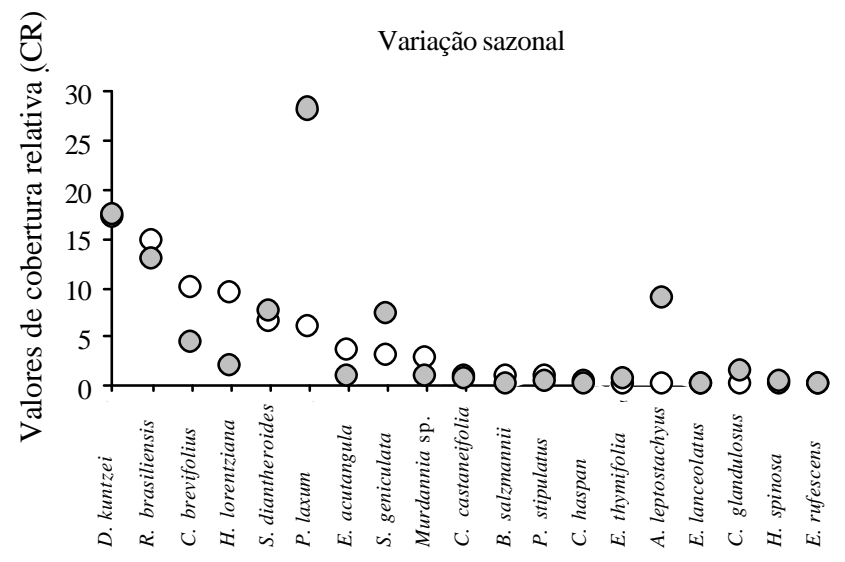

Figura 9. Variação sazonal do valor de cobertura relativa (CR) das espécies ocorrentes em ambos os períodos, chuvoso e de estiagem, campo inundável, Fazenda Nossa Senhora Aparecida, Pantanal de Poconé, MT, Brasil. $\bigcirc=$ Período chuvoso; $\bigcirc=$ Período de estiagem.

campos inundáveis, sendo que algumas espécies, entre elas Diodia kuntzei e Hyptis lorentziana, mantiveramse crescendo em ambas as fases, com marcado dimorfismo durante as fases do ciclo hidrológico. $\mathrm{Na}$ fase seca Panicum laxum, Setaria parviflora e Reimarochloa acuta foram as espécies dominantes naquelas áreas, com cobertura estimada em 50\%.

Prado et al. (1994) citaram Diodia kuntzei, Hyptis lorentziana, Panicum laxum e Reimarochloa brasiliensis como espécies muito abundantes.

O ano de 2001 foi mais seco, pois apesar das chuvas, não houve inundação pronunciada na área de estudo devido a baixa pluviosidade dos meses de dezembro (2000), janeiro e fevereiro (2001) (Fig. 3). Este fato também foi verificado por Soriano \& Alves (2003) que registraram meses considerados chuvosos (janeiro, fevereiro e março) com baixa precipitação durante o ano de 2001 para o Pantanal de Nhecolândia. No presente estudo isso refletiu na pequena cobertura e/ou não ocorrência de espécies exclusivamente aquáticas, como por exemplo, as Pontederiáceas, Ninfeáceas, Cabombáceas, comuns nas áreas de campos inundados (Pott \& Pott 2000; Schessl 1999; Pott \& Adamoli 1999; Prado et al. 1994).

Muitas gramíneas exóticas foram trazidas para o Brasil como forrageiras, principalmente da África, e espalharam-se por grandes extensões causando impactos sobre as comunidades campestres naturais, tendo como exemplo, as reservas criadas para proteger o Cerrado (Pivello 1992 apud Pivello et al. 1999). No campo estudado não se verificou a invasão de gramíneas exóticas, apesar de algumas espécies estarem presentes nas áreas adjacentes em relevos mais elevados. Estas espécies exóticas são eliminadas dos campos inundáveis por não suportarem o estresse hídrico anual da inundação. A única espécie introduzida e problemática no Pantanal é a Brachiaria subquadripara (Trin.) Hitchc., encontrada nas comunidades aquáticas no Parque Nacional do Pantanal (Pott et al. 2001), que, entretanto, não ocorreu nesta região de estudo.

Muitas espécies são ditas "invasoras", ou indesejáveis do ponto de vista do pecuarista, pois apesar de serem nativas, competem com as espécies forrageiras. Estão presentes na área de estudo Combretum lanceolatum, Croton glandulosus, Richardia grandiflora, Hydrolea spinosa e Axonopus leptostachyus, sendo esta última considerada invasora problemática em algumas regiões do Pantanal de Abobral (Allem \& Valls 1987).

Concluindo, o campo inundável estudado é caracterizado pela presença das seguintes espécies: $R$. brasiliensis, P. laxum, S. geniculata, A. leptostachyus, C. brevifolius, E. acutangula, B. myriophilloides, D. kuntzei, H. lorentziana, C. glandulosus, S. diantheroides e Murdannia sp., todas frequientes e com altos valores de cobertura relativa. A natureza da perturbação sazonal gera uma sucessão cíclica de espécies que se alternam entre espécies terrestres e aquáticas. Algumas espécies são comuns às duas fases, sendo denominadas de anfíbias. O pulso da umidade contribui para diversidade florística destas formações, sendo sua manutenção extremamente importante, devendo as fases, aquática e terrestre, serem analisadas como lados de uma mesma comunidade.

\section{Agradecimentos}

Ao Projeto Ecologia do Pantanal (Programa SHIFT), à FAPEMAT e ao Programa de Pósgraduação em Ecologia e Conservação da Biodiversidade da UFMT-IB, pelo apoio financeiro e logístico; ao CNPq, pela concessão de bolsa DTI; aos Dr. Arnildo Pott e Dra. Vali Pott, pelas identificações das espécies; à Dra. Hélida H. Bruno e Dr. Roberto Silveira, pelas sugestões e correções; ao Sr. Helio e Vantonildo e a equipe do Projeto, pelo auxílio no campo; ao proprietário da Fazenda Nossa Senhora Aparecida, Sr. Carlos Maciel (Sr. Titi). Os dados deste trabalho foram apresentados ao PPECB-UFMT para a obtenção do título de mestre. 


\section{Referências bibliográficas}

Allem, A.C. \& Valls, J.F.M. 1987. Recursos forrageiros nativos do Pantanal Mato-Grossense. Brasília, EMBRAPA/ CENARGEM, Documento 8.

Barbour, M.G.; Burk, J.H. \& Pitts, W.D. 1980. Terrestrial plant ecology. Pp. 156-182. California, The Benjamin/Cummings Publishing Company Inc.

Braun-Blanquet, J. 1979. Fitosociologia. Bases para el estudio de las comunidades vegetales. $\mathrm{H}$. Blume Ediciones, 17.

Cabido, M.; Acosta, A.; Diaz, S. \& Gonzalez, A.C. 1996. Factores estruturadores en paztizales cerranos del centro de Argentina. Pp.103-134. In: G. Sarmiento \& M. Cabido (eds.). Biodiversidad y Funcionamiento de Pastizales y Sabanas en América Latina. Mérida, CYTED/CIELAT.

Crispim, S.M.A; Santos, S.A.A.; Chalita, L.V.A.S.; Fernandes, A.H.B.M. \& Silva, M.P. 2002. Variação sazonal na frequiência e composição botânica em área de máxima inundação, Pantanal-MS, Brasil. Archivos de Zootecnia51: 149-160.

Cronquist, A. 1981. An integrated system of classification of flowering plants. New York, Columbia University Press.

Cunha, N.G. 1980. Considerações sobre os solos da subregião da Nhecolândia, Pantanal Mato-grossense. Corumbá, EMBRAPA/UEPAE

Grime, J.P. 1979.Plant Strategies and Vegetation Processes. Chichester, Wiley.

Hill, M.O. 1979. DECORAMA. A FORTRAN program for detrended correspondence analysis and reciprocal averaging. Ecology and Systematics. Ithaca, Cornell University.

Junk, W.J.; Bayley, P.B. \& Sparks, R.S. 1989. The flood pulse concept in river-floodplain systems. Pp. 110-127. D.P. Dodge (ed.). In: Proceedings of the International Large River Symposium (LARS). Can Spec Publ Fish Aquat Sci 106: 110-127.

Junk, W.J. \& Silva, C.J. 1999. O conceito do pulso de inundação e suas implicações para o Pantanal de Mato Grosso. Pp.17-28. In: Anais do II Simpósio sobre Recursos Naturais e Sócio-econômicos do Pantanal, Manejo e Conservação. Corumbá-MS, 1996. Brasília, EMBRAPACPAP.

Kent, M. \& Coker, P. 1994. Vegetation Description and Analysis. A Practical Approach. Chichester, UK. John Willey.

Kershaw, K.A. 1978. Quantitative and dinamic plant ecology. Edward Arnold.

Kiehl, K.; Eischeid, I.; Gettner, S. \& Walter, J. 1996. Impact of different sheep grazing intensities on salt marsh vegetation in northern Germany. Journal of Vegetation Science 7: 99-106.

Köppen, G.W. 1948. Climatologia. México, Buenos Aires, Ed. Fundo de Cultura Econômica.

Kovach, W.L. 1993. MVSP (Multivariate Statistical Package), Kovach PLC.

Krebs, C.J. 1985. Ecologia. Estúdio de la distribuición y la abundancia. México, Harla S.A.

Maarel, E. van der. 1993. Some remarks on disturbance and its relations to diversity and stability. Journal of Vegetation Science 4: 733-736.
Magurram, A.E. 1988. Ecological diversity and its measurement. Princeton, Princeton University Press.

Nogueira, F.; Couto, E.G. \& Bernardi, C.J. 2002, Geostatistics as a tool to improve sampling and statistical analysis in Wetlands: A Case Sudy on Dymanics of Organic Matter Distribution in the Pantanal of MatoGrosso, Brazil . Brazilian Journal of Biology 62(4B): 861-870.

Nunes da Cunha, C. \& Junk, W.J. 1999. Composição florística de capões e codilheiras: localização das espécies lenhosas quanto ao gradiente de inundação no Pantanal de Poconé, MT-Brasil. Pp.387-405. In: Anais do II Simpósio sobre Recursos Naturais e Sócio-econômicos do Pantanal, Manejo e Conservação. Corumbá-MS 1996. Brasília, EMBRAPA-CPAP.

Nunes da Cunha, C. \& Junk, W.J. 2001. Distribuition of Wood Plant Communities along the Flood Gradient in the Pantanal of Poconé, Mato Grosso, Brazil. International Journal of Ecology and Environmental 27: 63-70.

Nunes da Cunha, C.; Vilhalva, D.A.A. \& Ferreira, H. 2001. Espécies de Campo Inundável e de Brejo, Fazenda Retiro Novo, Pantanal de Poconé, MT (Lista preliminar). Pp. 1-14. In: Anais do III Simpósio sobre Recursos Naturais e Sócio-econômicos do Pantanal - Os desafios do novo milênio. Corumbá-MS 2000. Brasília, EMBRAPA-CPAP. CD-ROM.

Nunes da Cunha, C. \& Junk, W.J. 2004. Year-to-year changes in water level drive the invasion of Vochysia divergensinPantanal grasslands. Applied Vegetation Science 7 : 103-110.

Pakarinen, P. 1984. Cover estimation and sampling of Boreal vegetation in Northern Europe. Pp. 33-44. In: R. Knapp (ed.). Sampling methods and taxon analysis in vegetation science. Handbook of vegetation science, part 4. The Hague, Dr. W. Junk Publishers.

Palmer, M.W. 1994. Variation in species richness: toward a unification of hypotheses. Folia Geobotanica Phytotax 29: 511-530.

Peet, R.K. 1974. The measurement of species diversity. Annual Review of Ecology and Systematics 5: 285-307.

Pivello, V.R.; Carvalho, V.M.C.; Lopes, P.F.; Peccinini, A.A. \& Rosso, S. 1999. Abundance and distribution of native and alien grasses in a "Cerrado" (Brazilian Savanna) Biological Reserve. Biotropica 31(1): 71-82.

Ponce, V.M. 1995. Hidrologic and enviromental impact of the Paraná-Paraguay waterway on the Pantanal of Mato Grosso, Brazil. San Diego State University.http://ponce.sdsu.edu/ hidrovia_report.html (Acesso em 26/05/04).

Pott, A. 1986. Pastagens no Pantanal. Pp. 413-421. In: Anais do Congresso Brasileiro e Pastagens e $8^{\circ}$ Simpósio Sobre Manejo de Pastagens. Piracicaba, Publicação Fundação de Estudos Agrários Luiz de Queirós - ESALQ.

Pott, A. 1988. Pastagens no Pantanal. Corumbá EMBRAPA, CPAP, documentos 7.

Pott, A. \& Pott, V.J. 1994. Plantas do Pantanal. Corumbá, EMBRAPA-CPAP.

Pott, A. \& Adámoli, J. 1999. Unidades de vegetação do Pantanal dos Paiaguás. Pp. 183-202. In: Anais do II Simpósio sobre Recursos Naturais e Sócio-econômicos do Pantanal, Manejo e Conservação. Corumbá-MS 1996. Brasília, EMBRAPA-CPAP. 
Pott, A. \& Pott, V.J. 1999. Flora do Pantanal - Listagem de fanerógamas. pp.297-335. In:Anais do II Simpósio sobre Recursos Naturais e Sócio-econômicos do Pantanal, Manejo e Conservação. Corumbá-MS 1996. Brasília, EMBRAPA-CPAP.

Pott, A.; Nunes da Cunha, C.; Pott, V.J.; Silveira, E.A. \& Sartori, A.L.B. 2001. Avaliação ecológica Rápida - Parque Nacional do Pantanal e RPPNs do Entorno.

Pott, V.J. \& Pott, A. 2000. Plantas Aquáticas do Pantanal. Corumbá, EMBRAPA-CPAP.

Pozer, C.G. \& Nogueira, F. 2004. Pastagens nativas inundáveis da região norte do Pantanal de Mato Grosso: variações de biomassa e produtividade primária. Brazilian Journal of Botany 64(4): 859-866.

Prado, A.L.; Heckman, C.W. \& Martins, F.R. 1994. The seasonal succession of biotic communities in wetlands of the tropical wet-and-dry climatic zone: II. The aquatic macrophyte vegetation in the Pantanal of Mato Grosso, Brazil. Internatertionale Revue gesamten Hydrobiologie 79(4): 569-589.

Raunkier, C. 1934. The Life Forms of Plants and Statistical Plant Geography. Oxford, Clarendon Press.
Rizzini, C.T. Tratado de fitogeografia do Brasil: aspectos ecológicos, sociológicos e florísticos. $2^{\mathrm{a}} \mathrm{ed}$. São Paulo, ÂmbitoCultural.

Sala, O.E.; Oesterheld, M.; Léon, R.J.C. \& Soriano, A. 1986. Grazing effects upon plant community structure in subhumid grasslands of Argentina. Vegetatio67: 27-32.

Sarmiento, G. \& Monasterio, M. 1983. Life forms and fenology. Pp.79-108. In: F. Bourliére (ed.). Ecosystems of the Word: Tropical Savannas. Amsterdam, Elsevier.

Schessl, M. 1999. Floristic composition and structure of floodplain vegetation in northern Pantanal of Mato Grosso, Brasil.Phyton 39(2): 303-336.

Silva, C.J.; Watzen, K.M.; Nunes da Cunha, C. \& Machado, F.A. 2001. Biodiversity in the Pantanal wetland, Brasil. Pp.187-215. In: B. Gopal; W.J. Junk \& J.A. Davis (eds.). Biodiversity in wetlands: assessment, function and conservation. v.2. The Netherlands, Backnuys Publishers, Leiden.

Soriano, B.M.A. \& Alves, J.M. 2003. Boletim agrometeorológico ano 2001 para a sub-região da Nhecolândia, Pantanal, Mato Grosso do Sul, Brasil. Corumbá, EMBRAPA-CPAP.

Whittaker, R.H. 1975. Communities and Ecosystems . $2^{\text {nd }}$ ed., New York, Macmillian Publishing CO, Inc. 